\title{
Instability of flow past a cascade of circular cylinders
}

Link to publication record in Manchester Research Explorer

\section{Citation for published version (APA):}

Gajjar, J. S. B. (2006). Instability of flow past a cascade of circular cylinders. In Fluid Mechanics and its Applications/Fluid Mech. Appl. (Vol. 78, pp. 189-194). Springer Nature.

\section{Published in:}

Fluid Mechanics and its Applications|Fluid Mech. Appl.

\section{Citing this paper}

Please note that where the full-text provided on Manchester Research Explorer is the Author Accepted Manuscript or Proof version this may differ from the final Published version. If citing, it is advised that you check and use the publisher's definitive version.

\section{General rights}

Copyright and moral rights for the publications made accessible in the Research Explorer are retained by the authors and/or other copyright owners and it is a condition of accessing publications that users recognise and abide by the legal requirements associated with these rights.

\section{Takedown policy}

If you believe that this document breaches copyright please refer to the University of Manchester's Takedown Procedures [http://man.ac.uk/04Y6Bo] or contact uml.scholarlycommunications@manchester.ac.uk providing relevant details, so we can investigate your claim.

\section{OPEN ACCESS}




\title{
INSTABILITY OF FLOW PAST A CASCADE OF CIRCULAR CYLINDERS
}

\author{
J.S.B. Gajjar \\ School of Mathematics, \\ University of Manchester, Oxford Road, Manchester M13 9PL, U.K. \\ gajjar@manchester.ac.uk
}

Keywords: Stability, Cylinder, Navier-Stokes.

\section{INTRODUCTION}

The unsteady dynamics of laminar separation bubbles such as those arising in bluff body flows, or behind obstacles, is of considerable practical interest. Bubble formation near the leading edge of an aerofoil can dramatically alter the characteristics of the flow over the wing and induce stall. Similarly, laminar separation near the trailing edge of an aerofoil and instability of the separation bubble can influence the dynamics of the wake flow. The flow past a row bluff bodies placed in a uniform stream, is often used to model engine inlet flows, and thus an understanding of the unsteady dynamics of the eddies which form behind the bluff bodies and the unsteady development of the wake flow is useful in predicting the flow impinging on subsequent rotor blades.

There have been a number of recent studies aimed at investigating the instability of bubbles and wakes by using an idealised model for the mean flow. For example, in [1] a family of modified Falkner-Skan profiles is used to generate velocity profiles containing regions of reverse flow and the linear stability of these model profiles is then examined using the Orr-Sommerfeld equation. Based on their findings, Hammond and Redekopp [1] suggest that the flow in a separation bubble will be absolutely unstable and likely to trigger a global mode instability if the the extent of the reverse flow within the bubble approaches about $30 \%$ of the free stream value. Whilst such conclusions are interesting, as the authors point out, their analysis is based on the parallel flow assumption. The mean flow in a separation bubble is highly non-uniform and cannot be taken as being non-parallel. Work by Davies \& Carpenter[2] have

189

R. Govindarajan (ed.), Sixth IUTAM Symposium on Laminar-Turbulent Transition, 189-194. (C) 2006 Springer. Printed in the Netherlands. 
shown that in rotating disk flow, the inclusion of non-parallel terms can give rise to different conclusions as to whether a flow is absolutely or convectively uunstable.

Numerical simulations of separation bubbles in a boundary layer by Alam \& Sandham [3] also conclude that a considerable region of reverse flow is required for the flow in the bubble region to be absolutely unstable. In this work, the linear stability analysis is based on the Orr-Sommerfeld equation with a model velocity profile fitted to the numerically generated data. Again, the highly non-parallel nature of the mean flow raises serious questions about the validity of the stability analysis employed.

In the experimental work of $[4,5]$ the flow past a flat plate placed normal to an oncoming uniform flow, and with a splitter plate aligned with the flow direction, is studied. Hudy \& Naguib [4] suggest that under certain conditions, an absolute instability associated with the flow in the middle of the separation region may be responsible for the low frequency 'flapping' of the shear layer.

Recently Castro [6] has looked at the stability of the wake flow generated by a row of flat plates placed normal to the oncoming stream. Computations of the unsteady Navier-Stokes equations indicate a global instability, and the conclusions are further supported by linear Orr-Sommerfeld stability calculations of model velocity profiles fitted to the numerical data. Whilst the latter stability analysis is questionable, the numerical evidence from the time integrated Navier-Stokes equations is more convincing.

In the present study we consider an infinite row of circular cylinders placed in a uniform stream. The techniques that we have adopted to study the instability involve solving the two-dimensional eigenvalue problem, as in Cliffe $\&$ Tavener [7]. Once the mean flow is computed, the stability analysis is conducted by looking for perturbations proportional to $e^{-\beta t}$, where $\beta$ is a complex frequency, and $t$ is non-dimensional time. This leads to the solution of a generalised eigenvalue problem.

In what follows, we describe the problem formulation and give brief details of the numerical method. Some sample mean flow results are discussed. The results of the stabilty problem were felt to be too preliminary to include and will be published later when fully tested and validated.

\section{PROBLEM FORMULATION AND NUMERICAL TECHNIQUES EMPLOYED}

Consider an infinite row of cylinders placed in a uniform stream with $U_{\infty}$ in the $x$-direction. The centre of the cylinders lie on the $y$-axis at $x=0$, see figure 1 .

We consider the unsteady Navier-Stokes equations, with lengthscales nondimensionalised with respect to the cylinder radius, and velocities with $U_{\infty}$. The 


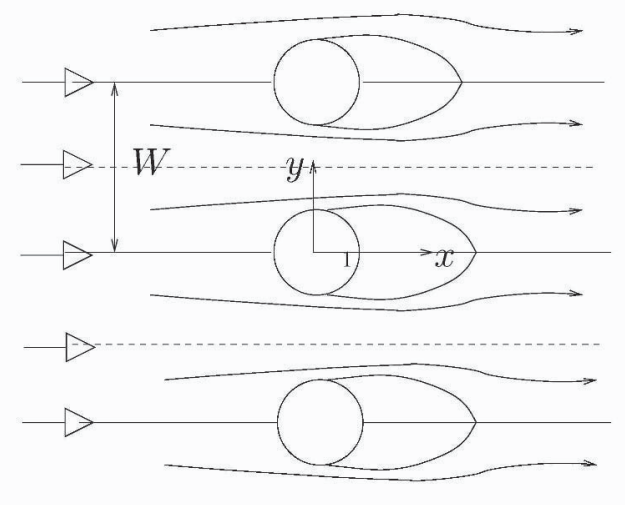

Figure 1. Sketch of flow past a cascade of circular cylinder
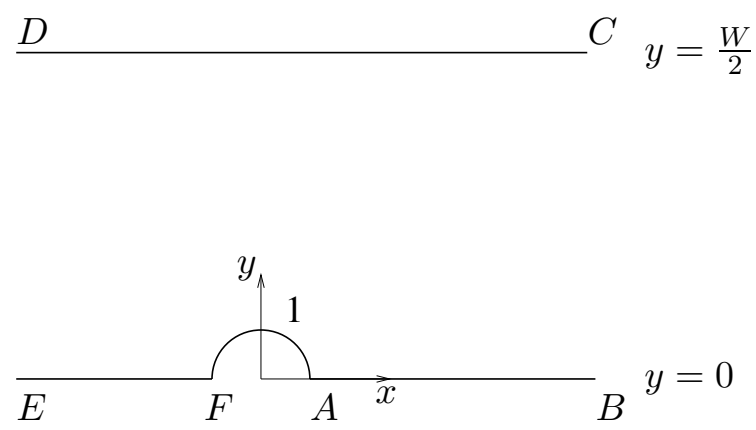

Figure 2. Sketch of the physical domain

equations written in streamfunction-vorticty formulation are:

$$
\begin{aligned}
\omega_{t}+\psi_{y} \omega_{x}-\psi_{x} \omega_{y} & =\frac{2}{R} \nabla^{2} \omega \\
\nabla^{2} \psi+\omega & =0
\end{aligned}
$$

Here $\psi$ is the streamfunction, $\omega$ the vorticity, and $R=U d / v$, where $U$ is the uniform speed relative to the cylinder at large distances from the cylinder, $d$ the diameter of the cylinder and $v$ is the kinematic viscosity of the fluid, and $t$ is non-dimensional time. With refernce to figure 1 , the non-dimensional gap width between the cylinders is $W$.

Let $\psi_{B}(x, y), \omega_{B}(x, y)$ denote the steady mean flow. In computing the mean flow, we exploit the symmetry in the problem by computing only in the region as shown in figure 2 . 
Perturbations to the mean flow are introduced as follows with

$$
\psi(x, y, t)=\psi_{B}(x, y)+e^{-\beta t} \tilde{\psi}(x, y), \quad \omega(x, y, t)=\omega_{B}(x, y)+e^{-\beta t} \tilde{\omega}(x, y)
$$

and after substituting into $(1,2)$ we linearise for small pertubations $\tilde{\psi}, \tilde{\omega}$. The perturbation equations are linearised using the same techniques as used for computing the mean flow with high-order finite differences in the $x$-direction and chebychev collocation in the other direction. For further details of the computation of the mean flow, see [8]. Finally the resulting eigenvalue problem for determining $\beta$ is of the form

$$
\mathbf{A} \mathbf{z}=\beta \mathbf{B} \mathbf{z} .
$$

This is the generalised eigenvalue problem. The eigenvalue problem here is not dissimilar to that arising in other contexts such as in the finite Taylor problem for the flow in the annular region between two concentric cylinders. Methods for the solution of (4) are discussed in Cliffe et al. [9], and we have adopted similar procedures. The main objective is to determine the eigenvalue spectrum for $\beta$ with the two parameters, the Reynolds number $R$ and gap-width $W$. Standard results demonstrate that the basic flow is stable if $\operatorname{Re}(\beta)>0$ for all eigenvalues of (4). We are particularly interested in determining what happens to the eigenvalues when the parameters change leading to loss of stability when a real eigenvalue crosses the imaginary axis, or when a complex conjugate pair crosses the imaginary axis leading to a Hopf bifurcation.
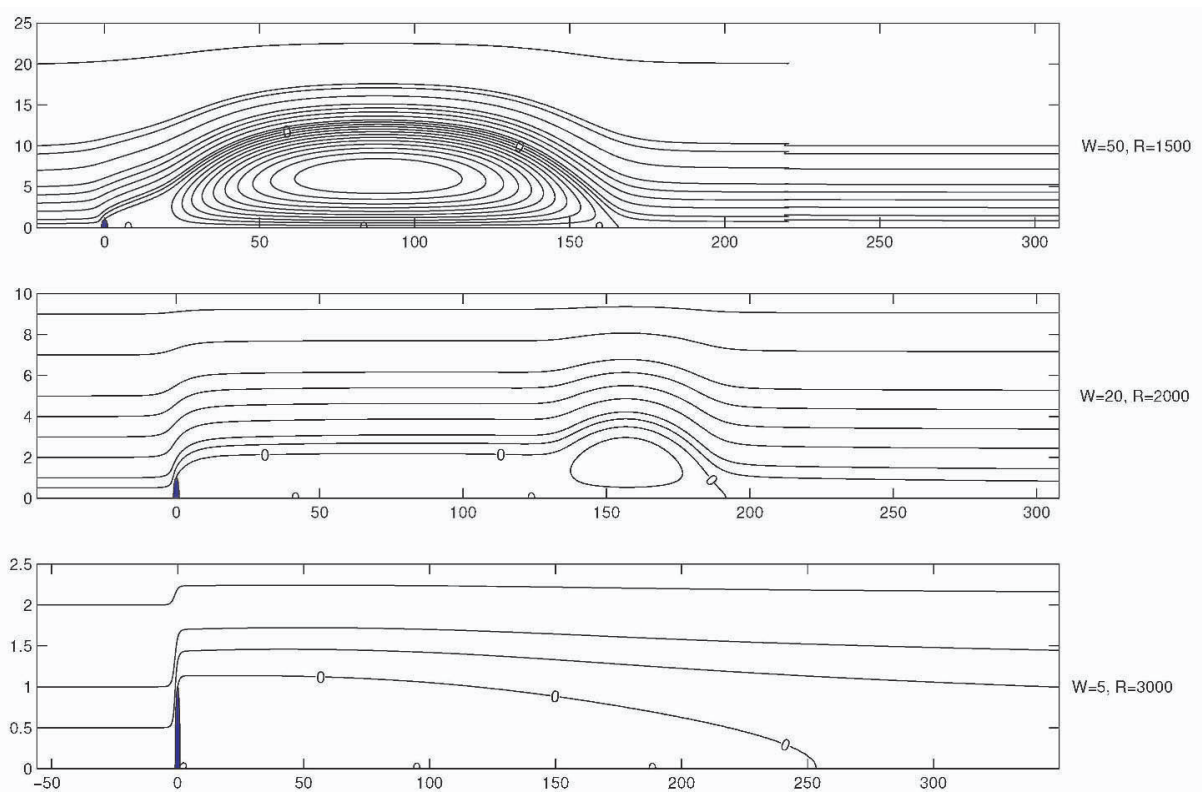

Figure 3. Contours of the stream function for gap widths $W=5,20$ and 50 at selected values of the Reynolds number 


\section{RESULTS}

The results for the steady flow have been extensively discussed in Gajjar $\&$ Nazzam [8]. The solution properties can be categorised into three distinct types, depending mainly on value of the gap width $W$ between the cylinders. For small values of the gap width the flow which develops in the wake is of boundary layer type with a long eddy forming behind the cylinder, see figure 3 , with the vorticity being non-uniform in the eddy region. For moderate gap widths the solution properties change dramatically particularly near the reattachment region. The solution, see figures 3,4 , comprise a long thin eddy followed by a much shorter fatter eddy near reattachment. The vorticity in the eddy near reattachment is uniform suggesting that the eddy is dominated by inviscid dynamics. Finally for large gap widths the flow in the wake is dominated by a very large eddy which initially grows linearly in size with increasing Reynolds numbers. For very large Reynolds numbers the eddy width eventually reaches some limiting value, although the length of the eddy is still linearly proportional to the Reynolds number, see figure 5.

Further comments and comparisons of the results with previous numerical and theoretical work for the steady flow is extensively documented in [8].
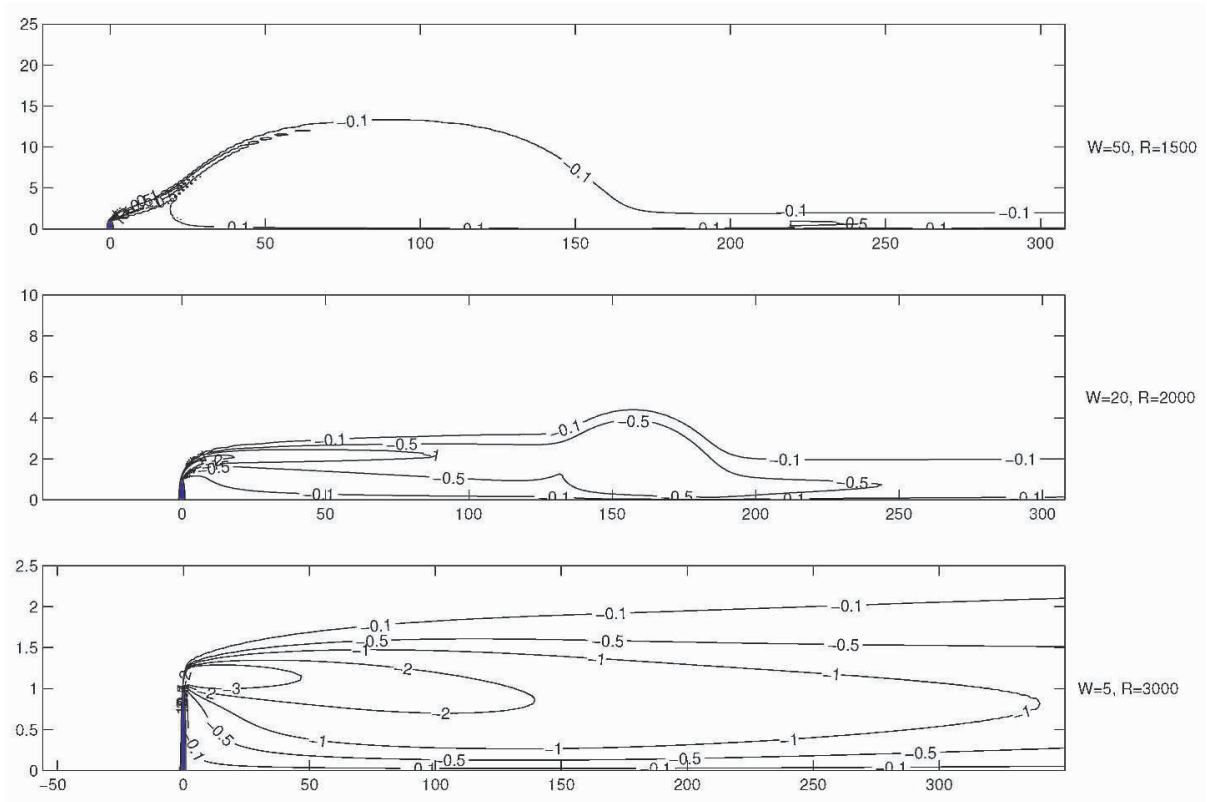

Figure 4. Contours of the vorticity function for gap widths $W=5,20$ and 50 at selected values of the Reynolds number 

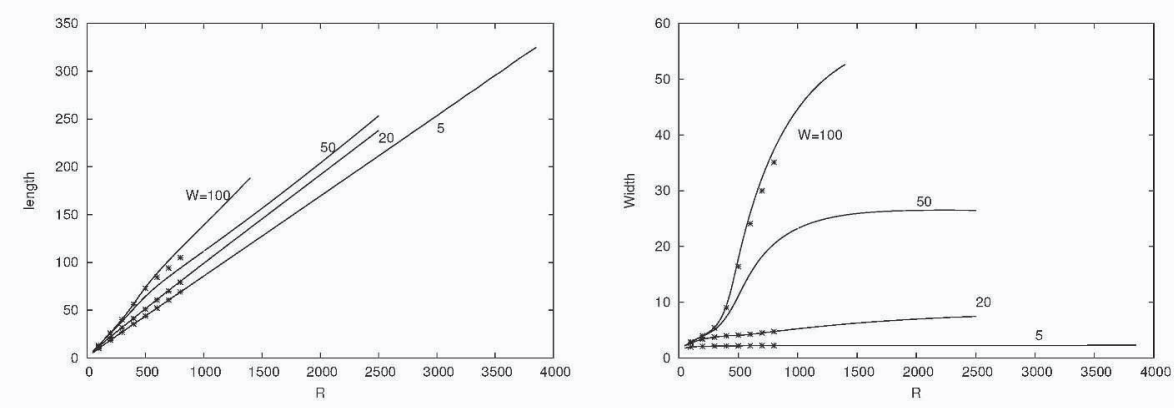

Figure 5. A plot of the eddy length and eddy width versus Reynolds number $R$ for gap widths of $W=5,20,50,100$. The symbols represent the data points of Fornberg [10]

[8] Gajjar, J.S.B. and Azzam, N., (2004). "Numerical solution of the Navier-Stokes equations for the flow in a cylinder cascade,” J.Fluid Mech., 520, 51-82.

[9] Cliffe, K.A., Garratt, T.J. and Spence, A., (1993). "Eigenvalues of the discretised NavierStokes equations with application to the detection of Hopf bifurcations," Adv. Comp. Maths, 1, 337-356.

[10] Fornberg, B., (1991). "Steady Incompressible Flow Past a Row of Circular Cylinders", J. Fluid Mech., 225, 655-671.

Finally concering the stability of the flow, our results are still at a preliminary stage and therefore not discussed here.

The author is grateful to the Royal Society of London for a travel grant to enable him to attend the meeting.

\section{REFERENCES}

[1] Hammond, D.A. and Redekopp, L.G. (1998). "Local and global instability properties of separation bubbles," Eur. J. Mech. B/Fluids, 17. 145-164.

[2] Davies, C. and Carpenter, P.W., (2003). "Global behaviour corresponding to the absolute instability of the rotating disc boundary layer", J. Fluid Mech., 486, 287-329.

[3] Alam, M. and Sandham, N.D., (2000). "Direct numerical simulation of 'short' laminar separation bubbles with turbulent reattachment," J. Fluid Mech., 410, 1-28.

[4] Hudy, L.M. and Naguib, A.M., (2003). "Wall-pressure measurements beneath a separating/reattaching flow region," Phy. Fluids, 15(3), 706-717.

[5] Cherry, N.J., Hillier, R. and Latour, M.P., (1984). "Unsteady measurements in a separated and reattaching flow,” J. Fluid Mech., 144, 13.

[6] Castro, I.P, (2004). "Stability of separated wakes," submitted to J. Fluid Mech.

[7] Cliffe, K.A., and Tavener S.J., (2004). "The effect of cylinder rotation and blockage ratios on the onset of periodic flows,", J. Fluid Mech., 501, 125-133. 